\title{
Characterization of Automotive High Voltage Networks for EMI Measurements
}

\author{
Dipl.-Ing. Martin Reuter ${ }^{1}$, Prof. Dr.-Ing. Stefan Tenbohlen ${ }^{1}$, Dr.-Ing. Wolfgang Köhler ${ }^{1}$, \\ ${ }^{1}$ Institut für Energieübertragung und Hochspannungstechnik, Universität Stuttgart, Germany, \\ *Martin.Reuter@ieh.uni-stuttgart.de
}

\begin{abstract}
One major task in developing electric cars is to control electromagnetic interferences (EMI) of their power train. Fast slew rates in power electronics increase efficiency, but also emitted radio frequency (RF) disturbances. One main approach to reduce EMI within automotive systems is a complete shielding of its high voltage (HV) power network. The shielding of $H V$ power networks changes the system configuration from the used unshielded single core multi wire harness into a coaxial three conductor layout, and therefore the characteristic impedances of the entire power network. Recent research shows that line impedance stabilization networks (LISNs) for substitution of low voltage power networks (according to CISPR 25), are not ideal to measure conducted emissions of HV components because of changed characteristic impedances and additional shielding.

This paper deals with a method of determining the high-frequency impedances of automotive HV power networks (300 kHz-200 MHz). A Vector Network Analyzer (VNA) is used to measure Scattering-Parameters of different HV power cables. Matrix conversions allow calculating an impedance network, which is able to represent automotive $H V$ networks.
\end{abstract}

\section{Introduction}

Increasing electrification of the automotive power train requires a new electrical power bus system. Recent automotive low voltage (LV) networks (12 V) are not able to meet power demands of electric driving engines because of high currents needed for power transmission. Automotive high voltage (HV) power networks, having voltage levels of $42-900 \mathrm{~V}$ but typically between $120-300 \mathrm{~V}$, reduce needed currents to values that can be handled more efficiently.

Maximum efficiency of power electronics requires fast slew rates of switching semiconductor devices. But fast slew rates result in bus disturbances, consisting of radiofrequency (RF) spectral components with high amplitudes. These RF disturbances may propagate along HV power lines, and are able to interfere with other electronic devices. Filters are a very strong element for suppression of conducted electromagnetic emissions (EME), but effective filter design requires knowledge about characteristic impedances of devices under test (DUT) and connected HV networks [1].

To ensure system's EMI performance, CISPR 25 defines an artificial network (AN/LISN), which represents a normalized automotive LV network with a standardized input impedance [2]. Every automotive component is tested in its EMI performance with this network. But devices connecting LV and HV network, e.g. DC/DC couplers, have to be terminated at their HV side connectors as later in their vehicle environment. Basically the LV LISNs can be used as HV termination, even shielding can be taken into consideration. However, this paper shows that wave impedances of HV cables differ substantially of the LV network characteristic impedance.

HV network impedance knowledge therefore is necessary for an effective HV filter design and to enable component EMI measurements with a realistic line impedance representing a vehicle environment.

In this paper, a method is presented to characterize automotive HV network impedances to simulate a HV vehicle environment. This simulation of a real vehicle environment is a basic requirement for comparable EMC measurements that allow to estimate their later performance mounted in an electric vehicle.

\section{LISNs for conducted EMI testing}

In 1983 a method was developed to measure conducted emissions of automotive components [3]. A Line Impedance Stabilization Network (LISN) was presented that provided mainly three functions:

1. Defined input impedance emulating a vehicle LV power network

2. Supply DUT with DC power

3. Impedance matching network to measurement system and decoupling of ambient distortions

This LISN was developed with aid of impedance measurements at automotive LV networks having two conductors, battery plus (BP) and battery minus (BM), which 
usually is grounded to autobody on its LV bus far end. Figure 1 shows a common two LISN EMI test setup.

Trying to use this LISN for HV component measurements two essential barriers occur: HV shielding and changed network input impedance.

In order to reduce emitted RF radiation of $\mathrm{HV}$ power electronics a complete shielding of the HV system is used. As in 1983 there was no need for any shielding of electrical power systems, the $\mathrm{AN}$ is not intended to emulate a shielded system. For security reasons HV systems are assembled floating, thus BM is not grounded. This directly leads to a three conductor system, whereas the emulated system of the CISPR 25 is a two conductor system.

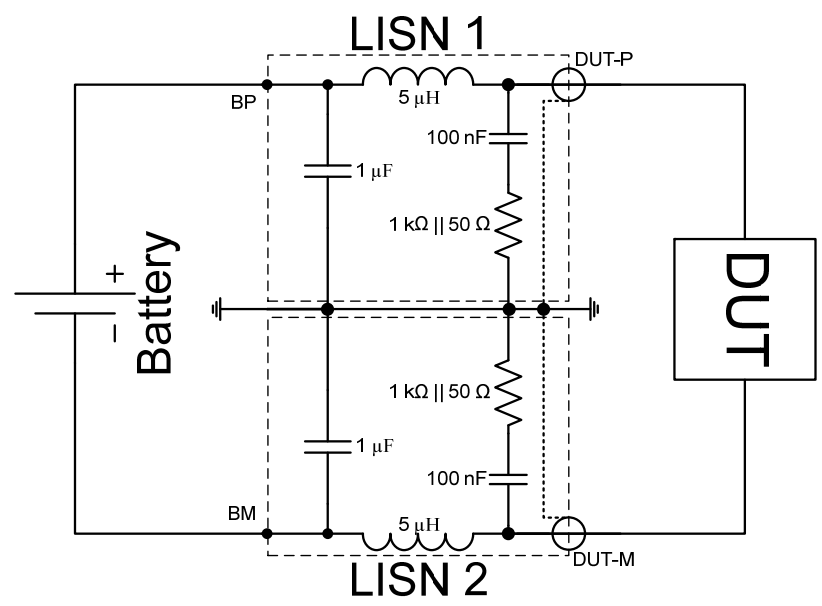

Figure 1

EEC of a two LISN setup for LV automotive component conducted EMI tests

By inserting two LISNs into a shielded box, connecting BP to one AN, BM to the second and Ground to the HV cable shields the changed conductor layout can be taken into consideration (see fig. 1). This leads to an input impedance of $100 \Omega$ between BM and BP and of $50 \Omega$ between each conductor to ground. As the characteristic wave impedances of the coaxial $\mathrm{HV}$ cables is significantly lower than $50 \Omega$ there is a mismatching of these transmission lines and standing waves and resonances can occur; hence it is not possible to evaluate the EMI performance of a $\mathrm{HV}$ component as in its vehicle environment.

Recent electric driven cars usually have their electric machine situated in the front motor area and an accumulator battery in the back, connected by 3-4 m of HV cable. Emitted RF power is sourced by fast switching power electronics, which is placed near the electric machine in the front. Conducted EMI can be coupled in cable wires (LV and $\mathrm{HV}$ ), and are able to interfere with connected systems. But these cable wires can also act as antennas and emitted RF radiation may also disturb galvanically isolated systems as sensitive radio antenna preamplifiers. The radiated RF power of long cable harnesses overbalances radiated EMI of the device itself (mainly housing) in the investigated frequency range. Thus for EMI performance of the complete system its conducted RF power on these long cables is essential [1].

Conducted emissions on automotive LV buses can be measured using ANs according to CISPR 25. If these ANs are used to emulate HV networks, disturbing RF sources of HV components are loaded with impedances of LV networks, which are different to the load impedances as later in their automotive HV environment. This measurement error cannot be corrected, as source impedances of RF sources (switching power semiconductors) cannot be determined. A network, emulating automotive HV networks as close as possible, would load HV components as in their vehicle environment and enables correct EMI performance measurements. This network should simulate 3-4 m of HV cable with attached accumulator battery, as every $\mathrm{HV}$ component is connected to this minimum network. More complex networks can be partitioned and reduced to presented minimum HV network.

\section{HV cable measurements}

For investigation of their RF behavior, six different automotive HV cables are examined with a Vector Network Analyzer (VNA). This instrument is used to measure Stray- or S-Parameters of electronic circuits and networks. Main high frequency attribute of a cable is its characteristic or wave impedance. Its line inductivity and capacity to ground also impact on the propagation of RF power. This chapter shows how to calculate these values of measured S-Parameters.

\subsection{Devices under Test $-\mathrm{HV}$ cables}

Table 1 introduces six different HV cables, which were investigated for presented results. All cables, except cable 1 , are of the same manufacturer. Cable 6 is not a coaxial type shielded wire (as the others are), this cable has a common shield for both conductors.

Table 1 Investigated HV cables and their assembly

\begin{tabular}{|l|l|l|}
\hline & $\varnothing$ & Shielding type \\
\hline Cable 1 & $25 \mathrm{~mm}^{2}$ & Single wire shield, screening foil \\
\hline Cable 2 & $25 \mathrm{~mm}^{2}$ & Single wire shield, screening foil \\
\hline Cable 3 & $25 \mathrm{~mm}^{2}$ & Single wire shield, no foil \\
\hline Cable 4 & $35 \mathrm{~mm}^{2}$ & Single wire shield, screening foil \\
\hline Cable 5 & $50 \mathrm{~mm}^{2}$ & Single wire shield, screening foil \\
\hline Cable 6 & $2 \times 6 \mathrm{~mm}^{2}$ & Common shield, screening foil \\
\hline
\end{tabular}

All cables are lengthened to $\ell=1 \mathrm{~m}$ and connected via low impedance cable glands to brass adapter boxes for N-Type RF connections to the measurement system as shown in figure 2. To have maximum analogy to vessel situation two cables of the same type are arranged parallel to each other.

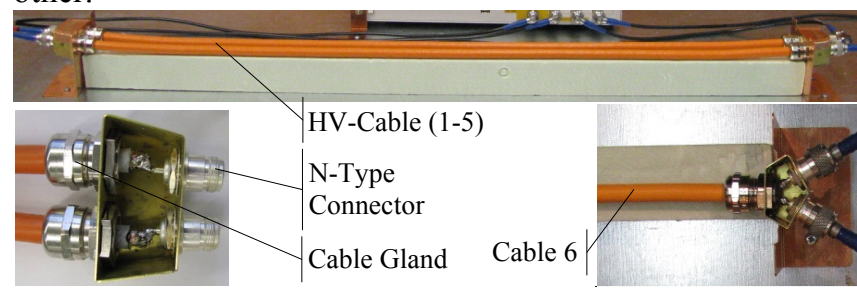

Figure 2
Photography of HV cable assembly with brass adapter boxes and low impedances cable glands 


\subsection{Measurement setup}

The S-Parameters of the HV cables are measured with an Agilent ENA E5070B network analyzer in a frequency range of $\mathrm{f}=300 \mathrm{kHz}-200 \mathrm{MHz}$. The sweep consists of 1601 points (logarithmic sweep). A mechanical calibration kit is used. The cables are situated in a height of $\mathrm{h}=5 \mathrm{~cm}$ above a conductive, grounded plane (figure 3 ). The coaxial type singular shielded cables have a spatial distance of $\mathrm{d}=4.25 \mathrm{~cm}$ (wires of cable 6 are spaced by assembly).

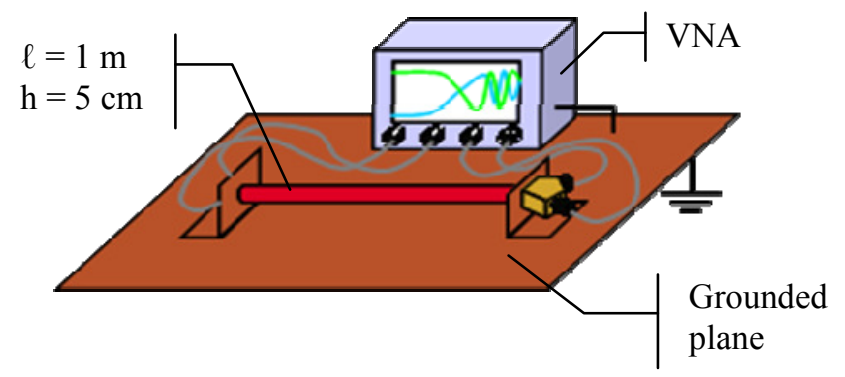

Figure 3 Measurement setup of HV cable 6 with VNA to determine RF behavior (SParameters).

\subsection{Characterization methodology}

S-Parameters are the quotient of reflected/transmitted wave to incident wave amplitudes. They are usually measured in the $50 \Omega$ system and can easily be converted to Z-, Y-, H- and T-Parameters. But they do not lead directly to the characteristic attributes of the measured transmission line. Characteristic cable values like wave impedances, line inductivities, ground capacitances or dielectric constants need to be calculated in further steps, which are presented in following chapters.

\subsubsection{Adaptor Deembedding}

Unfortunately the measurement of S-Parameters has to be carried out using special adapter boxes. These adapter boxes (see figure 2) are needed for reproducible measurements, to have best analogy to car situation and for the connection to measurement equipment (here: ENA5070B). The inevitable measurement error can be subtracted by

$$
\left[T_{\text {Cable }}\right]=\left[T_{\text {Adapter }}\right]^{-\frac{1}{2}} \cdot\left[T_{\text {Measurement }}\right] \cdot\left[T_{\text {Adapter }}\right]^{-\frac{1}{2}}
$$

Where $\left[\mathrm{T}_{\text {Cable }}\right]$ represents the transmission stray parameters of the $\mathrm{HV}$ cable, $\left[\mathrm{T}_{\text {Adapter }}\right]$ the T-Parameters of a doubled adapter box without $\mathrm{HV}$ cable in between and [ $\left.\mathrm{T}_{\text {Measurement }}\right]$ represents the committed measured T-Parameters. Via matrix conversion these T-Parameters are retransformed into S-Parameters.

\subsubsection{Calculation of line impedances}

For an impedance characterization these S-Parameters need to be connected to a given electric network. An electric equivalent circuit (EEC) of these cables can be found in figure 4. The simplest EEC of a transmission line is a T-
Network [1]. As the DUT consists of two parallel coaxial conductors a horizontally mirrored T-Network is needed, as shown in figure 4.

Figure 4

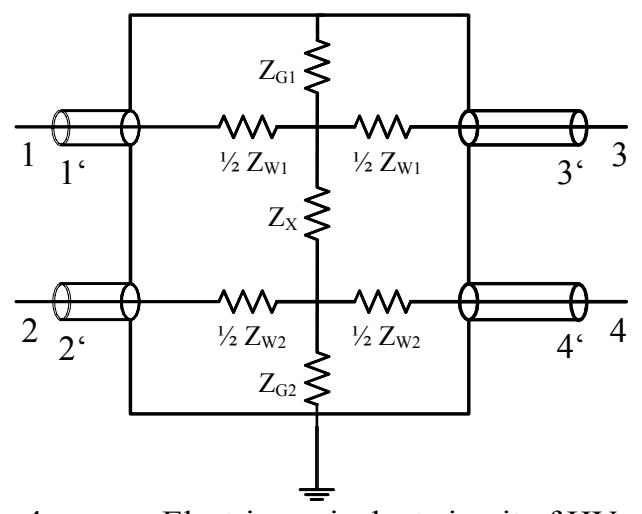

Electric equivalent circuit of HV cable, with conductor 1 between ports 1-3, conductor 2 between ports 2-4 and cross coupling impedance $\mathrm{Z}_{\mathrm{X}}$.

Calculating the Z-Parameters of this $1^{\text {st }}$ order, horizontally mirrored T-Network leads to an equation system of 5 different equations having 5 unknowns $\left(Z_{\mathrm{W} 1}, Z_{\mathrm{W} 2}, Z_{\mathrm{X}}, \mathrm{Z}_{\mathrm{G} 1}\right.$, and $Z_{\mathrm{G} 2}$ ). Inverting this equation system to the unknowns delivers

$$
\begin{aligned}
& Z_{W 1}=2 Z_{11}-2 Z_{31} \\
& Z_{W 2}=2 Z_{22}-2 Z_{42} \\
& Z_{X}=\frac{Z_{31}-Z_{42}}{Z_{12}}-Z_{12} \\
& Z_{G 1}=\frac{Z_{12}^{2}-Z_{31} \cdot Z_{42}}{Z_{12}-Z_{42}} \\
& Z_{G 2}=\frac{Z_{12}^{2}-Z_{31} \cdot Z_{42}}{Z_{12}-Z_{31}}
\end{aligned}
$$

The measured S-Parameters are transformed into ZParameters and inserted in this equation system.

An example for cable 1 is shown in figure 5.

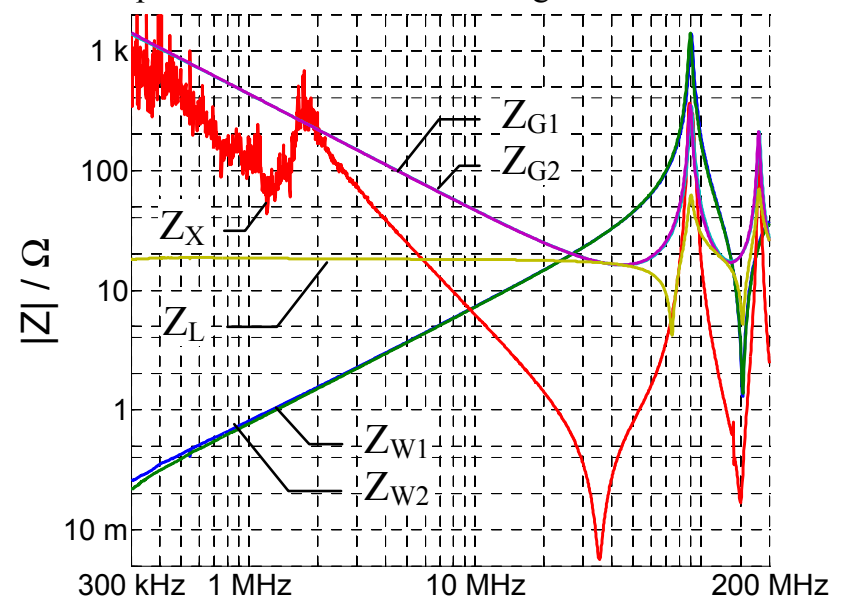

Figure 5 Values of HV cable 1 equivalent circuit passive resistances vs. frequency 
Figure 5 shows that the through resistances $Z_{\mathrm{W} 1}$ and $Z_{\mathrm{W} 2}$ start at a relatively small value $(150 \mathrm{~m} \Omega)$, increase linearly until approx. $50 \mathrm{MHz}$ and begin to oscillate above that. The resistances of inner conductor to grounded shielding $\left(\mathrm{Z}_{\mathrm{G} 1} \& \mathrm{Z}_{\mathrm{G} 2}\right)$ decrease linearly from $1 \mathrm{k} \Omega$ with increasing frequency and also oscillate above $50 \mathrm{MHz}$. Figure 5 also shows that both conductors act very similarly, as the curves of $Z_{\mathrm{W} 1} / Z_{\mathrm{W} 2}$ and $Z_{\mathrm{G} 1} / Z_{\mathrm{G} 2}$ are nearly without distinction; they are even equal for most of the investigated frequency range. The cross coupling resistance $Z_{X}$ does not have a clear frequency dependant behavior, but also oscillates in $\mathrm{RF}$ range. There are two clearly distinguishable frequency ranges of the line impedances: From $300 \mathrm{kHz}$ to approximately $30 \mathrm{MHz}$ the cable impedances have characteristic frequency dependencies, whereas above an oscillating behavior can be noticed. This is due to the quotient of wavelength to cable length: At $\lambda(\mathrm{f}) / \ell<6$ the cable is in quasi-stationary mode, acting like a network of concentrated elements, and at $\lambda(\mathrm{f}) / \ell \geq 6$ its transmission line characteristics overbalance and lead to this oscillating behavior [1].

\subsubsection{Impedance Interpretation}

The first resonance of the HV cable shown in figure 4 can be calculated as first maximum of $Z_{\mathrm{W}_{1}}$ (see figure 5). The resonance is occurring at $1=\lambda / 2=1 \mathrm{~m}$. In this example $\mathrm{f}_{\mathrm{r}}=84.4 \mathrm{MHz}$, meaning that at this frequency $\lambda=2 \mathrm{~m}$. In air an electromagnetic wave at $\mathrm{f}=84.4 \mathrm{MHz}$ has $\lambda=\mathrm{c}_{0} / \mathrm{f}=3.55 \mathrm{~m}$. This wave length shortening effect can be traced to the isolation material dielectric constant $\varepsilon_{\mathrm{r}}$, which can be calculated as

$$
\varepsilon_{r}=\left(\frac{c_{0}}{2 \ell \cdot f_{R 1}}\right)^{2}
$$

In the quasi stationary region of the frequency range, line and ground impedances have linear frequency dependencies. In this range line impedances act inductively and ground impedances capacitive. The line inductivity and ground capacity can be calculated via

$$
L_{S}=\frac{Z_{W 1}}{2 \pi f}
$$

$$
C_{G}=\frac{1}{2 \pi f \cdot Z_{G 1}}
$$

There are two different ways of calculating wave or characteristic impedance of a transmission line [4]:

$$
\begin{aligned}
& Z_{L}=\sqrt{Z_{11}^{2}-Z_{31}^{2}} \\
& Z_{L}=\sqrt{\frac{L_{\text {Serial }}}{C_{\text {Parallel }}}}
\end{aligned}
$$

Both formulas should lead to the same result, and can therefore be used to verify calculations.

Figure 6 shows an example for a graphical impedance analysis.

\subsection{Measurement results}

Table 1 lists the measured HV cable characteristics. It shows, that coaxial type shielded HV cables have a wave impedance in a range of $6.2-18.3 \Omega$, with direct dependency of their geometrical properties.

Table 1 Calculated transmission line attributes of automotive HV cables

\begin{tabular}{|l|c|c|c|c|c|c|c|}
\hline & $\begin{array}{c}\boldsymbol{\varnothing} \\
{\left[\mathbf{m m}^{2}\right]}\end{array}$ & $Z_{L}$ & $L_{S}$ & $C_{G}$ & $\sqrt{\frac{L_{S}}{C_{G}}}$ & $f_{r}$ & $\varepsilon_{r}$ \\
\hline Cable 1 & 25 & $18,3 \Omega$ & $123 \mathrm{nH} / \mathrm{m}$ & $363 \mathrm{pF} / \mathrm{m}$ & $18,4 \Omega$ & $89,8 \mathrm{MHz}$ & 2,7819 \\
\hline Cable 2 & 25 & $11 \Omega$ & $70 \mathrm{nH} / \mathrm{m}$ & $575 \mathrm{pF} / \mathrm{m}$ & $11,0 \Omega$ & $84,4 \mathrm{MHz}$ & 3,1521 \\
\hline Cable 3 & 25 & $11 \Omega$ & $68 \mathrm{nH} / \mathrm{m}$ & $553 \mathrm{pF} / \mathrm{m}$ & $11,1 \Omega$ & $85,8 \mathrm{MHz}$ & 3,0465 \\
\hline Cable 4 & 35 & $14,7 \Omega$ & $102 \mathrm{nH} / \mathrm{m}$ & $466 \mathrm{pF} / \mathrm{m}$ & $14,8 \Omega$ & $84,8 \mathrm{MHz}$ & 3,1252 \\
\hline Cable 5 & 50 & $6,2 \Omega$ & $43 \mathrm{nH} / \mathrm{m}$ & $\begin{array}{c}1102 \\
\mathrm{pF} / \mathrm{m}\end{array}$ & $6,3 \Omega$ & $85,8 \mathrm{MHz}$ & 3,0465 \\
\hline Cable 6 & $2 \times 6$ & $19,5 \Omega$ & $120 \mathrm{nH} / \mathrm{m}$ & $268 \mathrm{pF} / \mathrm{m}$ & $21,1 \Omega$ & $88,0 \mathrm{MHz}$ & 2,8977 \\
\hline
\end{tabular}

Compared to usually estimated values of automotive low voltage cables $\left(\mathrm{L}_{\mathrm{s}} \approx 1 \mu \mathrm{H} / \mathrm{m}, \mathrm{C}_{\mathrm{p}} \approx 100 \mathrm{pF} / \mathrm{m}\right)$ these measurements show around $10-20$ times smaller line inductivities and 3-10 times higher ground capacity values. This leads to considerably smaller wave impedances of HV networks.

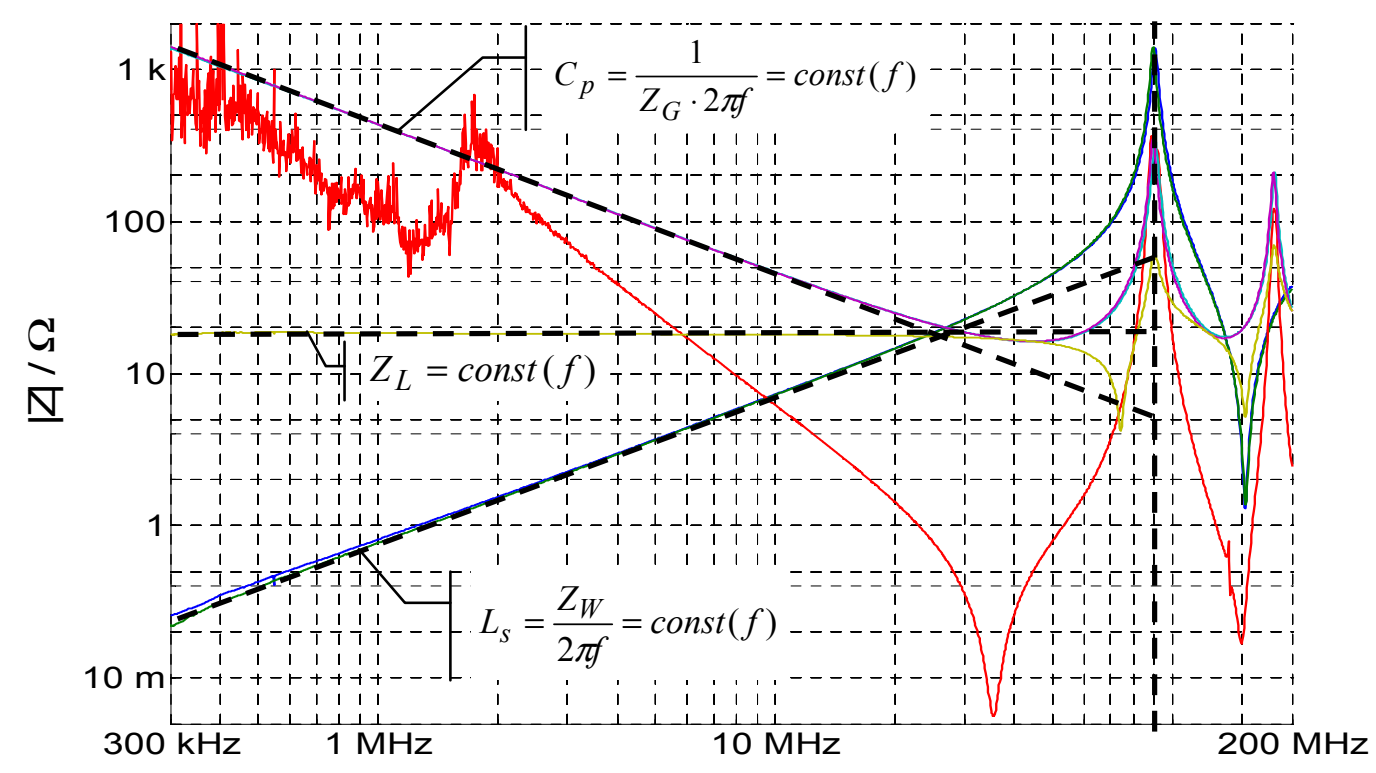

Figure 6

Graphical evaluations of cable 1 characteristic values $Z_{\mathrm{L}}, \mathrm{L}_{\mathrm{S}}, \mathrm{C}_{\mathrm{G}}$ and $\mathrm{f}_{\mathrm{r}}$. 
Due to their high line inductivity LV networks have relatively high line impedance in the frequency region of interest for EMC analysis. Additionally the transmission line character of $\mathrm{HV}$ cables is dominant above $10 \mathrm{MHz}$, where termination is defining its input impedance. Thus termination of HV cables becomes more important and has be taken into consideration, because it is determining the input impedance of a HV network in combination with cable characteristic impedance.

\section{$4 \quad$ Battery measurements}

As termination becomes important for the input impedance of a HV network, the HV batteries also need to be characterized. For nearly all fast switching power electronics the termination of extended $\mathrm{HV}$ cables is an HV accumulator battery. Therefore S-Parameters of such batteries need to be measured, and a $\pi$-EEC of the whole network calculated (consisting of 3-4 m HV cable and HV battery).

An automotive Li-Ion accumulator battery actually is under investigation, but measurement results will be presented soon.

\section{Conclusion}

This paper shows that HV network cables have a characteristic impedance considerably lower than $50 \Omega$. This leads to the perception that recently used LV LISNs for EMI testing of HV components imply a mismatch of input impedances. Optimized EMI performance of $\mathrm{HV}$ components using LV LISNs may not be reproduced mounted in car and could induce failed vehicle EMC tests. Thus further investigation about EMI testing of $\mathrm{HV}$ component conducted emissions is necessary. An AN could be developed, that matches closer the electromagnetic environment of $\mathrm{HV}$ components and test results compared to achieve an estimation of EMI performance on vehicle level. Knowledge of high frequency behavior of $\mathrm{HV}$ cables and batteries can be used to determine an electronic circuit, representing a minimum $\mathrm{HV}$ power network.

\section{Literature}

[1] Paul, Clayton R. (1992): „Introduction to Electromagnetic Compatibility“, New York: John Wiley \& Sons, Inc.

[2] IEC/CISPR 25:2002 / DIN-EN 55025:2003, "Radio disturbance characteristics for the protection of receivers used on board vehicles, boats and on devices Limits and methods of measurement", CENELEC European Committee for Electrotechnical Standardization, rue de Stassart 35, B-1050 Bruxelles.

[3] Yamamoto S., Ozeki, O. "RF Conducted Noise Measurements of Automotive Electrical and Electronic Devices Using Artificial Network", IEEE Trans. Veh. Technol., vol. VT-32, no. 4, pp. 247-253, Nov. 1983.
[4] Berroth, Manfred (2001): "Theorie der Schaltungen III / Theory of circuitry III", Lecture Notes, University of Stuttgart. 